\title{
Percepção dos usuários sobre o cuidado prestado por equipes participantes do Programa Nacional de Melhoria do Acesso e da Qualidade da Atenção Básica no Brasil*
}

Service users' perception about healthcare provided by teams participating in the National Program for Primary Care Access and Quality Improvement in Brazil

\section{Percepción de los usuarios sobre el cuidado prestado por los equipos participantes del Programa Nacional de Mejora del Acceso y la Calidad de la Atención Básica en Brasil}

\author{
Daisy Maria Xavier de Abreu' 1 - o orcid.org/0000-0002-6855-8612 \\ Lucas Henrique Lobato de Araújo' \\ Clarice Magalhães Rodrigues dos Reis ${ }^{1}$ \\ Ângela Maria de Lourdes Dayrell de Lima' \\ Alaneir de Fátima dos Santos ${ }^{2}$ \\ Alzira Oliveira Jorge ${ }^{2}$ \\ Délcio Fonseca Sobrinho² \\ Antônio Thomaz Gonzaga da Matta Machado² \\ 'Universidade Federal de Minas Gerais, Faculdade de Medicina, Belo Horizonte, MG, Brasil \\ ${ }^{2}$ Universidade Federal de Minas Gerais, Faculdade de Medicina, Belo Horizonte, MG, Brasil
}

\section{Resumo}

Objetivo: analisar a percepção dos usuários sobre o cuidado prestado por equipes participantes do Programa Nacional de Melhoria do Acesso e da Qualidade da Atenção Básica (PMAQ-AB) no Brasil. Métodos: estudo transversal com dados de entrevistas com usuários das equipes, em 2012. Empregou-se regressão logística multinomial para analisar a associação entre nível de percepção (muito bom/bom, regular, ruim/muito ruim) e variáveis de quatro atributos da atenção básica: acessibilidade, integralidade, longitudinalidade e coordenação do cuidado. Resultados: dos 65.391 entrevistados, 78,9\% avaliaram o atendimento como muito bom/bom, 19,0\% como regular e 2,1\% como ruim/muito ruim. A avaliação muito bom/bom foi associada a questões sobre os quatro atributos estudados. Destacam-se as associações com atendimento pelo mesmo médico (OR 3,21; $\left.\mathrm{IC}_{95 \%} 2,68 ; 3,83\right)$ e realização do exame físico na consulta $\left(\mathrm{OR} 3,08 ; \mathrm{IC}_{95 \%} 2,50 ; 3,79\right)$. Conclusão: a maioria dos usuários avaliou positivamente 0 atendimento, contudo existe percepção de problemas no cuidado conforme os atributos recomendados.

Palavras-Chave: Atenção Primária à Saúde; Satisfação do Paciente; Qualidade da Assistência à Saúde; Estudos Transversais.

* Agência financiadora: Ministério da Saúde - Processo n² 25000.183474/2011-70.

Endereço para correspondência:

Daisy Maria Xavier de Abreu - Av. Alfredo Balena, n 190, sala 727, Santa Efigênia, Belo Horizonte, MG. CEP: 30130-100

E-mail:dmxa@medicina.ufmg.br 


\section{Introdução}

A atenção primária à saúde no Brasil, eixo estruturante do Sistema Único de Saúde (SUS), é caracterizada por ser, de modo geral, o primeiro contato dos usuários com o sistema de saúde. ${ }^{1} \mathrm{~A}$ atenção primária à saúde (denominada no Brasil atenção básica à saúde - ABS) se pautou no arcabouço teórico-metodológico norteado pelos atributos essenciais de acessibilidade, longitudinalidade, integralidade e coordenação do cuidado. ${ }^{2-4}$ Entretanto, construir um modelo de atenção à saúde a partir destas premissas representa uma tarefa árdua e seu desenvolvimento exige a qualificação da atenção ofertada, devendo contar com instrumentos que possam avaliar a atuação das equipes responsáveis por este nível de atenção de forma permanente. ${ }^{5}$

Nesse aspecto, a experiência internacional tem indicado a necessidade de se considerar a contribuição do usuário no monitoramento técnico e social dos serviços de saúde, ${ }^{6,7}$ pois sua avaliação poderá agregar elementos que reforcem intervenções voltadas para a melhoria da qualidade dos serviços prestados.

\section{A experiência internacional tem indicado a necessidade de se considerar a contribuição do usuário no monitoramento técnico e social dos serviços de saúde, pois sua avaliação poderá agregar elementos que reforcem intervenções voltadas para a melhoria da qualidade dos serviços prestados.}

No Brasil, o interesse em avaliar a participação da comunidade nos processos de planejamento e avaliação, incorporando a questão da avaliação do usuário, ganhou força na década de 1990, sendo bastante expressiva a produção científica sobre 0 tema. ${ }^{8}$ Ainda que as abordagens sobre avaliação de usuários sejam bastante variadas e sujeitas a questionamentos acerca das estratégias metodológicas adotadas, os resultados dos estudos tendem a apontar elevado grau de satisfação dos usuários com o serviço recebido. ${ }^{8}$ Esta constatação deve ser avaliada face aos persistentes problemas no acesso e na qualidade dos serviços de saúde no Brasil. Trata-se, portanto, de uma temática merecedora de um aprofundamento sobre dimensões da assistência pres- tada que possam estar associadas ao resultado positivo da avaliação dos usuários sobre os serviços de saúde.

No âmbito da atenção básica, o Ministério da Saúde iniciou, a partir de 2011, uma política de monitoramento e avaliação dos serviços de saúde ofertados pela rede nacional de Atenção Básica - o Programa Nacional de Melhoria do Acesso e da Qualidade da Atenção Básica (PMAQ-AB). Este programa objetiva estabelecer um sistema de monitoramento e avaliação que contempla apoio técnico-científico para estimular a melhoria da qualidade do processo de trabalho das equipes de atenção básica, impulsionando os gestores locais a buscar estratégias de promoção de equidade e cobertura universal em saúde em seus territórios. ${ }^{9}$ Uma fase constitutiva do Programa é a avaliação externa, realizada por instituições de ensino e pesquisa com o objetivo de verificar in loco as condições de acessibilidade e de qualidade da atenção oferecida pelas equipes de ABS que aderiram ao Programa, a partir da observação direta e do registro do ponto de vista das próprias equipes e de seus usuários.

A base de dados gerada pelo PMAQ-AB para a avaliação da ABS representa uma oportunidade de se conhecerem, em âmbito nacional, aspectos da organização e do processo da assistência prestada, do ponto de vista dos usuários, a partir de sua experiência de uso, condições de acessibilidade, utilização de serviços de saúde e percepção sobre os serviços recebidos. Com isso, se agrega ao conhecimento existente sobre o tema uma discussão sobre dimensões do cuidado ofertado, ao se procurar identificar possíveis barreiras organizacionais para a acessibilidade e a qualidade da ABS do ponto de vista de quem efetivamente utiliza os serviços. Parte-se do pressuposto de que a avaliação do usuário depende do contexto em que a assistência à saúde é prestada, e também são sugeridas características estruturais que podem ser responsáveis por essa avaliação. ${ }^{8}$

Com base nessas reflexões, o objetivo do presente estudo é analisar a percepção dos usuários no que se refere aos serviços prestados por equipes participantes do PMAQ-AB, considerando-se os quatro principais atributos da ABS: acessibilidade, integralidade, longitudinalidade e coordenação do cuidado.

\section{Métodos}

Trata-se de um estudo transversal. Utilizaram-se os dados obtidos da avaliação externa das equipes aderidas ao PMAQ$-\mathrm{AB}$, realizada entre maio e dezembro de 2012, sob iniciativa 
do Departamento de Atenção Básica do Ministério daSaúde, em parceria com instituições de ensino superior do país.

No ano de 2012, cerca de 52\% das equipes de ABS aderiram ao PMAQ-AB e, na avaliação externa, foram visitadas e entrevistadas 17.479 equipes de ABS e 65.391 usuários, em 3.972 municípios brasileiros.

Foram analisadas informações do módulo III da avaliação externa do programa, obtidas por meio de questionário estruturado aplicado aos usuários presentes nas UBS no dia da visita das equipes de entrevistadores do PMAQ-AB. Os questionários foram aplicados por equipes de entrevistadores devidamente capacitadas para este fim pelas instituições de ensino e pesquisa responsáveis pela avaliação externa do PMAQ-AB. 0 objetivo dessas questões foi avaliar, na visão do usuário, as condições de acessibilidade, utilização de serviços de saúde e percepção sobre o cuidado recebido. ${ }^{9}$ Esse módulo foi aplicado a quatro usuários das EAB avaliadas, no dia da avaliação na UBS. A amostra intencional foi obtida a partir da aceitação de convite verbal. Os usuários que aceitaram participar da entrevista assinaram o Termo de Consentimento Livre e Esclarecido (TCLE). Como critérios de inclusão para seleção dos entrevistados, foram considerados: utilização do serviço nos últimos 12 meses; e entrevista de, no mínimo, uma mãe ou responsável por crianças menores de 2 anos e um(a) idoso(a).

Para a avaliação da percepção dos usuários sobre 0 cuidado prestado por equipes avaliadas pelo PMAQ-AB, utilizou-se o nível de percepção dos usuários, conforme a seguinte escala: muito ruim, ruim, regular, bom e muito bom. Essas respostas decorrem da questão: "Na sua opinião, o cuidado que o(a) senhor(a) recebe da equipe de saúde é". A variável dependente correspondeu à categorização dessas respostas em três categorias: muito bom/bom, regular e ruim/ muito ruim, sendo a última a categoria de referência.

As variáveis independentes se referem aos atributos essenciais da ABS, divididas em quatro atributos que serão descritos abaixo. Em parênteses, descrevem-se as opções de resposta oferecidas aos usuários entrevistados.

Acessibilidade: (i) presença de médico na unidade de saúde ou em atividades no território em todos os horários de funcionamento da UBS (sim ou não); (ii) o usuário consegue ser escutado para resolver qualquer problema, sem ter hora marcada (sim, sempre/sim, algumas vezes/ não); (iii) qual o tempo de espera para atendimento (0 a 15 minutos/15 a 30 minutos/acima de 30 minutos).

Integralidade: (i) a equipe busca resolver suas necessidades/problemas na própria unidade de saúde (sim/ não/algumas vezes); (ii) nas consultas, os profissionais da equipe fazem o exame físico (sim/na maioria das vezes/ quase nunca/nunca); (iii) os profissionais de saúde indagam sobre outras necessidades de saúde, além daquelas relacionadas com o motivo da consulta (sim/na maioria das vezes/quase nunca/nunca); (iv) os profissionais de saúde sugerem soluções adequadas a sua realidade (sim/ na maioria das vezes/quase nunca/nunca).

Longitudinalidade: (i) atendimento pelo mesmo médico ( $\mathrm{sim} / \mathrm{sim}$, algumas vezes/quase nunca/nunca); (ii) visita domiciliar de outros profissionais da equipe de saúde (sim/sim, algumas vezes/sim, quase sempre/não).

Coordenação do cuidado: (i) quando o usuário precisa, os profissionais da equipe conseguem marcar uma consulta com outros profissionais ou especialistas (sim/sim, algumas vezes/nunca); (ii) depois que foi atendido(a) por outros profissionais fora desta unidade de saúde, a equipe conversou sobre este atendimento (sim, sempre/sim, algumas vezes/não); (iii) há facilidade para saber dos resultados dos seus exames que chegam a esta unidade de saúde (sim, sempre/sim, algumas vezes/não).

Na etapa descritiva, calcularam-se frequências absolutas e relativas nas categorias das variáveis sociodemográficas dos participantes, a saber: sexo (masculino/feminino), idade (16-24 anos, 25-34 anos, 35-59 anos, 60 anos ou mais, seminformação), renda ( 0 a R \$ 500,00, de R \$ 501,00 a R \$ 999,00, de R \$1.000,00 a R \$ 9.999,00, sem informação), escolaridade (1 a 4 anos de estudo, 5 a 7 anos de estudo, 8 anos e mais de estudo), raça/cor da pele (branca, preta, amarela, parda/mestiça, indígena, sem informação).

Em uma segunda etapa analítica, foi realizada uma análise multivariável por regressão logística multinomial, utilizando-se o método de entrada Enter no Social Statistical Package for the Social Sciences (SPSS), versão 19. Foram excluídas as variáveis que apresentaram multicolinearidade e ausência de associação pelo teste qui-quadrado. A categoria ruim/muito ruim foi adotada como grupo de referência.

Utilizaram-se como variáveis de ajuste o sexo, a escolaridade, a faixa de renda e aidade dos usuários. Para indicar a significância estatística das associações, adotou-se o nível de significância de $5 \%$ ( $\mathrm{p} \leq 0,05)$ utilizando-se testes bicaudais. Estimaram-se as razões de odds (ou razões de chances) e respectivos intervalos de confiança (IC) de $95 \%$. A qualidade de ajuste dos modelos criados foi analisada pelo teste de Hosmer e Lemeshow. ${ }^{10} 0$ modelo final foi selecionado considerando-se um valor de $\mathrm{p}>0,05$ para o teste, 0 que corresponde a uma ausência de diferença estatisticamente 
significativa na distribuição dos valores dependentes efetivos e previstos. Também se avaliou o ajuste do modelo pelo $R^{2}$.

A base de dados utilizada foi disponibilizada pelo Departamento de Atenção Básica do Ministério da Saúde e o estudo teve a aprovação do Comitê de Ética em Pesquisa da Universidade Federal de Minas Gerais (UFMG) pelo parecer 28804 de 30/05/2012 (CAAE: 02396512.8.0000.5149).

\section{Resultados}

Dos 65.391 usuários das equipes de ABS aderidas ao PMAQ-AB em 2012, 77,7\% eram mulheres e 22,3\% homens. A maioria dos entrevistados tinha entre 35 e 59 anos (39,8\%). Em relação à raça/cor da pele, $44,8 \%$ se classificaram como pardos ou mestiços. A maior parte dos entrevistados tinha renda familiar entre $R \$ 500,00 \mathrm{e}$ $\mathrm{R} \$ 1.000,00$ (57,3\%), que, à época da coleta de dados, correspondiam a valores entre 1 e 2 salários mínimos, $\mathrm{e}$ 70,3\% tinham mais de 8 anos de escolaridade (Tabela 1 ).

A grande maioria dos usuários (78,9\%) avaliou 0 atendimento das equipes como bom ou muito bom, $19 \%$ como regular e $2,1 \%$ como ruim ou muito ruim.

Em relação ao atributo acessibilidade (Tabela 2), na análise ajustada, a variável "presença do médico na unidade ou em atividade no bairro em todos os horários de funcionamento" apresentou associação com a percepção do usuário como muito bom/bom (OR 4,$\left.29 ; \mathrm{IC}_{95 \%} 3,42 ; 5,38\right)$. No que se refere ao "tempo de espera dos usuários para atendimento", observou-se que, para espera entre 1 e 15 minutos e entre 16 e 30 minutos, o grau de associação com a avaliação de seu atendimento classificada como muito bom/bom foi semelhante (OR 1,80; $\mathrm{IC}_{95 \%} 1,36 ; 2,38$ e OR 1,82; $\mathrm{IC}_{95 \%}$ 1,$37 ; 2,45$, respectivamente). Verificou-se uma associação positiva entre os usuários que consideraram ser sempre escutados para resolver seus problemas mesmo sem hora marcada e o nível de percepção muito bom/bom (OR: 2,35; $\mathrm{IC}_{95 \%}$ 1,70; 3,23).

Nas questões relacionadas ao atributo integralidade (Tabela 3), observou-se que, para todas as variáveis analisadas, houve uma forte associação com a percepção positiva do usuário sobre o serviço. Assim, a associação entre a variável "se a equipe busca resolver as necessidades/problemas na própria unidade de saúde" e a avaliação muito bom/bom do usuário resultou em um OR de 28,68 (IC: 24,03; 34,22) e, mesmo que esta atenção tenha ocorrido somente algumas vezes, a associação apresentou um 0R 7,22 (IC: 6,12;8,53). Esta associação foi também observada para aqueles usuários que responderam como regular, mas com menor força.

Para a variável "nas consultas, os profissionais fazem exame físico", a associação foi positiva com a avaliação muito bom/bom para a frequência sempre (OR 3,08; $\left.\mathrm{IC}_{95 \%} 2,50 ; 3,79\right)$ e para na maioria das vezes (OR 2,12; $\left.\mathrm{IC}_{95 \%} 1,70 ; 2,63\right)$. As questões que tratam do interesse dos profissionais de saúde no que respeita a outras necessidades de saúde do usuário, além daquelas relacionadas com o motivo da consulta e a sugestão de soluções adequadas à realidade apresentaram também associação com a avaliação positiva dos usuários, sendo maior a intensidade quanto maior a frequência da atitude dos profissionais.

Quanto ao atributo longitudinalidade (Tabela 4), a variável "atendimento prestado pelo mesmo médico" teve uma associação positiva com a avaliação muito bom/ bom do usuário para quando esse atendimento é sempre prestado pelo mesmo médico $\left(\mathrm{OR} 3,21 ; \mathrm{IC}_{95 \%} 2,68 ; 3,83\right)$ e nos casos em que 0 atendimento ocorre algumas vezes pelo mesmo médico (OR 2,60; $\mathrm{IC}_{95 \%}$ 2,14; 3,29). Para a variável "visita domiciliar de outros profissionais da equipe de saúde", a associação foi positiva, com a avaliação muito bom/bom, tanto nos casos nos quais ela ocorre sempre (OR 3,60; $\left.\mathrm{IC}_{95 \%} 2,87 ; 4,50\right)$ quanto naqueles em que a visita ocorre algumas vezes (OR 3,43; $\left.\mathrm{IC}_{95 \%} 2,63 ; 4,48\right)$.

Para 0 atributo coordenação do cuidado (Tabela 5), houve associação positiva entre a variável "marcação da consulta com outros profissionais ou especialistas" e a avaliação do usuário como muito bom/bom (OR 5,80; $\left.\mathrm{IC}_{95 \%} 4,92 ; 6,84\right)$. A associação também é positiva quando "após 0 atendimento fora da unidade a equipe conversou sobre este atendimento" (OR 5,49; IC $\left._{95 \%} 4,28 ; 7,03\right)$, com a avaliação muito bom/bom. Em relação à variável "facilidade para saber dos resultados dos exames que chegam a esta unidade de saúde", a associação positiva foi elevada, com uma percepção considerada muito bom/bom, quando esta situação ocorreu sempre (OR 4,79) $\mathrm{IC}_{95 \%}$ $4,09 ; 5,60)$, e, para os que tiveram acesso aos exames algumas vezes, a força da associação foi menor (OR 2,13; $\left.\mathrm{IC}_{95 \%} 1,80 ; 2,55\right)$. Esta diferença ocorreu também para as variáveis "marcação de consulta com outros profissionais ou especialistas" e "após atendimento fora da unidade de saúde, a equipe conversou sobre este atendimento".

\section{Discussão}

A percepção dos usuários sobre o cuidado prestado pelas equipes no PMAQ-AB apresentou um percentual 
Tabela 1 - Características sociodemográficas dos usuários atendidos por equipes participantes do Programa Nacional de Melhoria do Acesso e da Qualidade da Atenção Básica,Brasil, 2012

\begin{tabular}{|c|c|c|}
\hline Variáveis & $\mathbf{n}$ & $\%$ \\
\hline \multicolumn{3}{|l|}{ Sexo } \\
\hline Homens & 14.600 & 22,3 \\
\hline Mulheres & 50.791 & 77,7 \\
\hline \multicolumn{3}{|l|}{ Idade (anos) } \\
\hline $16-24$ & 9.737 & 14,9 \\
\hline $25-34$ & 13.927 & 21,3 \\
\hline $35-59$ & 26.005 & 39,8 \\
\hline 60 ou mais & 14.844 & 22,7 \\
\hline Sem informação & 878 & 1,3 \\
\hline \multicolumn{3}{|l|}{ Renda (R\$) } \\
\hline 0 a 500 & 5.686 & 8,7 \\
\hline 501 a 999 & 37.472 & 57,3 \\
\hline 1.000 a 9.999 & 8.089 & 12,4 \\
\hline Sem informação & 14.144 & 21,6 \\
\hline \multicolumn{3}{|l|}{ Escolaridade } \\
\hline 1 a 4 anos & 16.295 & 24,9 \\
\hline 5 a 7 anos & 3.098 & 4,8 \\
\hline 8 anos ou mais & 45.998 & 70,3 \\
\hline \multicolumn{3}{|l|}{ Raça/cor da pele } \\
\hline Branca & 25.164 & 38,5 \\
\hline Preta & 7.966 & 12,2 \\
\hline Amarela & 1.735 & 2,7 \\
\hline Parda/mestiça & 29.311 & 44,8 \\
\hline Indígena & 616 & 0,9 \\
\hline Sem informação & 599 & 0,9 \\
\hline Total & 65.391 & 100,0 \\
\hline
\end{tabular}

elevado de avaliação positiva, pois mais de três quartos dos usuários entrevistados consideraram o nível de atenção recebida como muito bom/bom.

Além disso, para as questões abordadas sobre acessibilidade, integralidade, longitudinalidade e coordenação do cuidado, houve associação positiva entre os serviços prestados e a percepção dos usuários para uma avaliação muito bom/bom.

Estes resultados são importantes para a discussão sobre a percepção dos usuários quanto à acessibilidade e à qualidade da atenção básica no Brasil, pois parte de uma avaliação que aborda dimensões do cuidado para além de uma pesquisa mais geral sobre satisfação dos usuários. Isto porque, embora a avaliação dos usuários tenda a ser positiva, existe percepção de problemas na assistência recebida de acordo com os atributos recomendados para a atenção básica em saúde.
A percepção positiva dos usuários em relação ao serviço constatada nos resultados está em conformidade com o descrito em outros estudos, como em revisões bibliográficas e de conceitos sobre o tema, e em estudos como o realizado em Ribeirão Preto, em $2005,,^{8,11-13}$ o qual aponta que quem é atendido, em geral, indica uma boa avaliação do atendimento recebido.

Em termos de acessibilidade, a importância da presença do médico em todos os horários de funcionamento da UBS na avaliação positiva dos usuários pode ser entendida como uma demanda por garantia da atenção médica na ABS durante todo o período de atendimento. A Política Nacional de Atenção Básica preconiza que as UBS devem organizar o seu processo de trabalho de forma a combinar as jornadas de trabalho dos profissionais das equipes e os horários e dias de funcionamento, a fim de se garantir o maior acesso possível, o vínculo entre usuários e profissionais, a continuidade, a coordenação 


\section{Tabela 2 - Associação entre a percepção dos usuários sobre o cuidado prestado por equipes participantes do Programa Nacional de Melhoria do Acesso e da Qualidade da Atenção Básica e variáveis do atributo acessibilidade, Brasil, 2012}

\begin{tabular}{|c|c|c|c|c|c|c|c|c|}
\hline \multirow{4}{*}{ Variáveis } & \multicolumn{8}{|c|}{ Percepção } \\
\hline & \multicolumn{4}{|c|}{ Regular } & \multicolumn{4}{|c|}{ Muito bom/bom } \\
\hline & \multicolumn{2}{|c|}{ Análise bruta ${ }^{a}$} & \multicolumn{2}{|c|}{ Análise ajustada ${ }^{b}$} & \multicolumn{2}{|c|}{ Análise bruta ${ }^{\mathrm{a}}$} & \multicolumn{2}{|c|}{ Análise ajustadab } \\
\hline & $O R^{c}$ & $\mathrm{IC}_{95 \%}{ }^{\mathrm{d}}$ & $\mathrm{OR}^{\mathrm{C}}$ & $\mathrm{IC}_{95 \%}{ }^{\mathrm{d}}$ & $O R^{C}$ & $\mathrm{IC}_{95 \%}{ }^{\mathrm{d}}$ & $\mathbf{O R}^{\mathrm{c}}$ & $\mathrm{IC}_{95 \%}{ }^{\mathrm{d}}$ \\
\hline \multicolumn{9}{|c|}{ Presença do médico na unidade de saúde ou em atividades no bairro em todos os horários de funcionamento } \\
\hline Não & 1,00 & - & 1,00 & - & 1,00 & - & 1,00 & - \\
\hline Sim & $2,23^{e}$ & 1,$96 ; 2,54$ & $1,63^{e}$ & 1,$30 ; 2,06$ & $6,63^{\mathrm{e}}$ & 5,$85 ; 7,51$ & $4,29^{\mathrm{e}}$ & 3,$42 ; 5,38$ \\
\hline \multicolumn{9}{|c|}{ Consegue ser escutado para resolver qualquer problema, sem hora marcada } \\
\hline Não & 1,00 & - & 1,00 & - & 1,00 & - & 1,00 & - \\
\hline Sim, sempre & $0,73^{e}$ & 0,$62 ; 0,87$ & 1,25 & 0,$91 ; 1,74$ & 0,97 & 0,$82 ; 1,14$ & $2,35^{\mathrm{e}}$ & 1,$70 ; 3,23$ \\
\hline Sim, algumas vezes & $0,78^{e}$ & 0,$65 ; 0,95$ & 0,83 & 0,$62 ; 1,10$ & $0,58^{\mathrm{e}}$ & 0,$47 ; 0,69$ & $0,61^{\mathrm{e}}$ & 0,$46 ; 0,81$ \\
\hline \multicolumn{9}{|c|}{ Tempo de espera para atendimento (em minutos) } \\
\hline 31 ou mais & 1,00 & - & 1,00 & - & 1,00 & - & 1,00 & - \\
\hline 1 a 15 & $1,47^{e}$ & 1,$15 ; 1,89$ & 1,20 & 0,$90 ; 1,60$ & $3,56^{\mathrm{e}}$ & 2,$79 ; 4,54$ & $1,80^{\mathrm{e}}$ & 1,$36 ; 2,38$ \\
\hline 16 a 30 & $1,49^{e}$ & 1,$31 ; 1,96$ & 1,38 & 1,$03 ; 1,84$ & $2,45^{e}$ & 1,$87 ; 3,22$ & $1,82^{\mathrm{e}}$ & 1,$37 ; 2,45$ \\
\hline
\end{tabular}

Por regressão logística binomial, tendo como referência a categoria ruim/muito ruim da variável dependente e a primeira categoria de resposta da variável independente. Ajustada por idade, faixa de renda, sexo e escolaridade.

'OR: odds ratio (razão de chances).

${ }^{d} \mathrm{C}_{950 \%}:$ Intervalo de confiança de $95 \%$.

ep valor $\leq 0,05$

e a longitudinalidade do cuidado. ${ }^{1}$ A situação identificada, a partir da avaliação positiva dos usuários em relação à presença de médicos, deve ser analisada, particularmente, em municípios mais distantes, carentes e nas periferias de grandes centros, com a reconhecida dificuldade de fixação de médicos para 0 atendimento nos diferentes níveis de atenção e, especialmente, na ABS. Girardi et al. (2014) ${ }^{14}$ apontaram, em estudo sobre escassez de médicos no Brasil, desigualdades sociorregionais na distribuição de médicos, particularmente nas regiões Norte e Nordeste. A falta do médico possivelmente tem efeito sobre a opinião dos usuários, ${ }^{15}$ sendo que a avaliação tende a ser pior quando a UBS não dispõe de médico em todos os horários de funcionamento. Neste aspecto, o Programa Mais Médicos do Brasil (PMMB), criado com o objetivo de levar o profissional médico para áreas rurais, remotas e periféricas do país, é uma estratégia bem avaliada pelos usuários, no que tange à disponibilidade e à sensibilidade do profissional do Programa, o que induz a população a reafirmar a importância da continuidade do PMMB ${ }^{16}$

Em termos do acolhimento como categoria de acessibilidade, observou-se que o tempo de espera para atendimento teve forte associação com a avaliação do usuário. Assim como em outros estudos, ${ }^{17,18}$ constatou-se que quanto menor o tempo de espera maior a chance de o usuário estar satisfeito com o serviço prestado. Além disso, a possibilidade de o paciente ser escutado para resolver qualquer problema sem ter hora marcada também teve associação com uma avaliação mais positiva. Tais condições de acessibilidade parecem influenciar fortemente a avaliação do usuário, pois a espera prolongada foi identificada como um dos principais motivos de insatisfação dos usuários e, por outro lado, 0 interesse demonstrado na escuta do usuário tem sido identificado como um recurso para o estabelecimento do vínculo entre usuário e serviço de saúde. ${ }^{19}$

Para as questões concernentes à integralidade, houve uma manifestação também positiva dos usuários em relação àquelas analisadas. Em consonância com estudo desenvolvido em Porto Alegre ${ }^{20}$ os resultados da presente pesquisa apontam para uma forte associação entre a resolução de problemas pela equipe de ABS e a percepção do usuário. A integralidade da atenção passa pelas possibilidades de resolubilidade da equipe, a partir de um maior ou menor comprometimento desta em buscar respostas para os problemas dos usuários. Outro aspecto valorizado foi o interesse manifestado pela equipe sobre outras demandas de saúde do usuário. A associação encontrada com uma avaliação mais positiva parece indicar que, para os usuários, é importante, ademais, que as equipes busquem ampliar sua atuação 
Tabela 3 - Associação entre a percepção dos usuários sobre o cuidado prestado por equipes participantes do Programa Nacional de Melhoria do Acesso e da Qualidade da Atenção Básica e variáveis do atributo integralidade, Brasil, 2012

\begin{tabular}{|c|c|c|c|c|c|c|c|c|}
\hline \multirow{4}{*}{ Variáveis } & \multicolumn{8}{|c|}{ Percepção } \\
\hline & \multicolumn{4}{|c|}{ Regular } & \multicolumn{4}{|c|}{ Muito bom/bom } \\
\hline & \multicolumn{2}{|c|}{ Análise bruta ${ }^{a}$} & \multicolumn{2}{|c|}{ Análise ajustadab } & \multicolumn{2}{|c|}{ Análise bruta ${ }^{a}$} & \multicolumn{2}{|c|}{ Análise ajustadab } \\
\hline & $\mathbf{O R}^{\mathrm{c}}$ & $\mathrm{IC}_{95 \%}{ }^{\mathrm{d}}$ & $\mathbf{O R}^{\mathrm{c}}$ & IC $_{95 \%}{ }^{\mathrm{d}}$ & $\mathbf{O R}^{\mathrm{c}}$ & $\mathrm{IC}_{95 \%}{ }^{\mathrm{d}}$ & $\mathbf{O R}^{\mathrm{c}}$ & $\mathrm{IC}_{95 \%}{ }^{\mathrm{d}}$ \\
\hline \multicolumn{9}{|c|}{ A equipe busca resolver suas necessidades/problemas na própria unidade de saúde } \\
\hline Não & 1,00 & - & 1,00 & - & 1,00 & - & 1,00 & - \\
\hline Sim & $5,85^{\mathrm{e}}$ & 5,$05 ; 6,76$ & $4,81^{\mathrm{e}}$ & 4,$03 ; 5,74$ & $12,31^{\mathrm{e}}$ & 5,$23 ; 19,21$ & $28,68^{\mathrm{e}}$ & 24,$03 ; 34,22$ \\
\hline Algumas vezes & $3,61^{\mathrm{e}}$ & 3,$14 ; 4,16$ & $3,32^{\mathrm{e}}$ & 2,$82 ; 3,91$ & $9,31^{e}$ & 4,$51 ; 14,01$ & $7,22^{\mathrm{e}}$ & 6,$12 ; 8,53$ \\
\hline \multicolumn{9}{|c|}{ Nas consultas os profissionais da equipe fazem exame físico } \\
\hline Nunca & 1,00 & - & 1,00 & - & 1,00 & - & 1,00 & - \\
\hline Sim & $2,45^{\mathrm{e}}$ & 2,$03 ; 2,96$ & $1,51^{\mathrm{e}}$ & 1,$23 ; 1,86$ & $9,15^{\mathrm{e}}$ & 7,$62 ; 10,99$ & $3,08^{\mathrm{e}}$ & 2,$5 ; 3,79$ \\
\hline Na maioria das vezes & $2,02^{\mathrm{e}}$ & 1,$65 ; 2,48$ & $1,41^{\mathrm{e}}$ & 1,$13 ; 1,79$ & $3,98^{\mathrm{e}}$ & 3,$28 ; 4,84$ & $2,12^{\mathrm{e}}$ & 1,$70 ; 2,63$ \\
\hline Quase nunca & $1,33^{\mathrm{e}}$ & 1,$10 ; 1,60$ & $1,21^{e}$ & 0,$98 ; 1,46$ & $1,48^{\mathrm{e}}$ & 1,$16 ; 1,68$ & 1,21 & 0,$99 ; 1,49$ \\
\hline \multicolumn{9}{|c|}{ Os profissionais de saúde indagam sobre outras necessidades de saúde, além daquelas relacionadas com o motivo da consulta } \\
\hline Nunca & 1,00 & - & 1,00 & - & 1,00 & - & 1,00 & - \\
\hline Sim & $2,26^{\mathrm{e}}$ & 1,$94 ; 2,64$ & $1,32^{\mathrm{e}}$ & 1,$09 ; 1,61$ & $8,56^{\mathrm{e}}$ & 7,$37 ; 9,94$ & $2,61^{e}$ & 2,$51 ; 3,68$ \\
\hline Na maioria das vezes & $1,99^{\mathrm{e}}$ & 1,$63 ; 2,43$ & $1,30^{e}$ & 1,$03 ; 1,65$ & $4,78^{\mathrm{e}}$ & 3,$94 ; 5,80$ & $2,35^{\mathrm{e}}$ & 1,$83 ; 2,91$ \\
\hline Quase nunca & $1,22^{\mathrm{e}}$ & 1,$02 ; 1,47$ & 0,93 & 0,$75 ; 1,16$ & $1,52^{\mathrm{e}}$ & 1,$27 ; 1,82$ & 1,08 & 0,$87 ; 1,34$ \\
\hline \multicolumn{9}{|c|}{ Os profissionais de saúde sugerem soluções adequadas a sua realidade } \\
\hline Nunca & 1,00 & - & 1,00 & - & 1,00 & - & 1,00 & - \\
\hline Sim & $2,48^{\mathrm{e}}$ & 2,$12 ; 2,89$ & $1,66^{\mathrm{e}}$ & 1,$38 ; 2,03$ & $8,52^{\mathrm{e}}$ & 7,$32 ; 9,91$ & $3,04^{e}$ & 2,$51 ; 3,68$ \\
\hline Na maioria das vezes & $1,90^{\mathrm{e}}$ & 1,$60 ; 2,25$ & 1,32 & 0,$08 ; 1,61$ & $3,53^{\mathrm{e}}$ & 2,$99 ; 4,16$ & $1,77^{\mathrm{e}}$ & 1,$45 ; 2,16$ \\
\hline Quase nunca & $1,20^{\mathrm{e}}$ & 1,$00 ; 1,43$ & 1,10 & 0,$88 ; 1,36$ & $1,33^{e}$ & 1,$11 ; 1,58$ & 1,17 & 0,$95 ; 1,45$ \\
\hline
\end{tabular}

aPor regressão logística binomial, tendo como referência a categoria ruim/muito ruim da variável dependente e a primeira categoria de resposta da variável independente. ${ }^{b}$ Ajustada por idade, faixa de renda, sexo e escolaridade.

'OR:odds ratio (razão de chances).

${ }^{\mathrm{I}} \mathrm{C}_{95 \%}$ : intervalo de confiança de $95 \%$

ep valor $\leq 0,05$.

para além das queixas apresentadas, demonstrando uma preocupação também com um cuidado mais integral. ${ }^{19}$

Em relação à longitudinalidade, que engloba vínculo e responsabilização, abordada nas questões do atendimento pelo mesmo médico, acesso aos resultados de exames e visita domiciliar de outros profissionais, há também alguns tópicos merecedores de reflexão. Para os usuários, a disponibilidade destas formas de cuidado é percebida como positiva, pois a avaliação foi altamente positiva nestas condições. Como destacado na política da ABS, a resolutividade das ações realizadas depende do vínculo e da responsabilização das equipes estabelecidas com os usuários, bem como do significado que se dá à relação profissional/usuário. ${ }^{20}$

A coordenação do cuidado também tem impacto sobre a avaliação positiva dos usuários quanto à ABS. $\mathrm{Na}$ análise de associação, as questões avaliadas sobre este atributo indicaram que os usuários valorizam as ações que procuram promover a integração entre os níveis do sistema de saúde. ${ }^{21}$

Ainda que a avaliação possa ser considerada muito positiva para grande parte das questões avaliadas, não podem ser minimizadas algumas fragilidades identificadas na organização e no processo da assistência prestada. Conforme destacam Fausto et al. (2014), ${ }^{22}$ estas dimensões devem ser consideradas e avaliadas no conjunto de serviços ofertados na ABS.

Além de se evidenciar a percepção dos usuários, interessa identificar e investigar o que aparece de negativo ou com baixa avaliação, pois é o que revela o que não está sendo bem avaliado e o que precisa ser modificado. Nesta direção, os resultados encontrados oferecem indícios de que a acessibilidade, a integralidade, a longitudinalidade e a coordenação do cuidado devem servir também para que as equipes repensem sua forma de organização e o aperfeiçoamento do 
Tabela 4 - Associação entre a percepção dos usuários sobre o cuidado prestado por equipes participantes do Programa Nacional de Melhoria do Acesso e da Qualidade da Atenção Básica e variáveis do atributo longitudinalidade, Brasil, 2012

\begin{tabular}{|c|c|c|c|c|c|c|c|c|}
\hline \multirow{4}{*}{ Variáveis } & \multicolumn{8}{|c|}{ Percepção } \\
\hline & \multicolumn{4}{|c|}{ Regular } & \multicolumn{4}{|c|}{ Muito bom/bom } \\
\hline & \multicolumn{2}{|c|}{ Análise bruta ${ }^{a}$} & \multicolumn{2}{|c|}{ Análise ajustadab } & \multicolumn{2}{|c|}{ Análise bruta ${ }^{a}$} & \multicolumn{2}{|c|}{ Análise ajustadab } \\
\hline & $\mathbf{O R}^{\mathrm{c}}$ & IC $_{95 \%}{ }^{\mathrm{d}}$ & $O R^{c}$ & IC $_{95 \%}{ }^{\mathrm{d}}$ & $\mathbf{O R}^{\mathrm{c}}$ & IC $_{95 \%}{ }^{\mathrm{d}}$ & $\mathbf{O R}^{\mathrm{c}}$ & $\mathrm{IC}_{95 \%}{ }^{\mathrm{d}}$ \\
\hline \multicolumn{9}{|c|}{ Atendimento pelo mesmo médico } \\
\hline Nunca & 1,00 & - & 1,00 & - & 1,00 & - & 1,00 & - \\
\hline Sim & $2,08^{e}$ & 1,$74 ; 2,49$ & $1,77^{\mathrm{e}}$ & 1,$47 ; 2,13$ & $4,59^{\mathrm{e}}$ & 3,$87 ; 5,45$ & $3,21^{e}$ & 2,$68 ; 3,83$ \\
\hline Sim, algumas vezes & $2,18^{\mathrm{e}}$ & 1,$75 ; 2,70$ & $1,82^{\mathrm{e}}$ & 1,$47 ; 2,27$ & $3,75^{\mathrm{e}}$ & 3,$05 ; 4,62$ & $2,60^{e}$ & 2,$14 ; 3,29$ \\
\hline Quase nunca & $1,38^{\mathrm{e}}$ & 1,$13 ; 1,69$ & $1,26^{\mathrm{e}}$ & 1,$02 ; 1,55$ & $1,51^{e}$ & 1,$24 ; 1,83$ & $1,23^{e}$ & 1,$01 ; 1,50$ \\
\hline \multicolumn{9}{|c|}{ Visita domiciliar de outros profissionais da equipe de saúde } \\
\hline Não & 1,00 & - & 1,00 & - & 1,00 & - & 1,00 & - \\
\hline Sim, sempre & $1,41^{\mathrm{e}}$ & 1,$12 ; 1,77$ & $1,31^{\mathrm{e}}$ & 1,$05 ; 1,67$ & $4,05^{e}$ & 3,$25 ; 5,04$ & $3,60^{e}$ & 2,$87 ; 4,50$ \\
\hline Sim, algumas vezes & $1,88^{\mathrm{e}}$ & 1,$44 ; 2,46$ & $1,77^{\mathrm{e}}$ & 1,$47 ; 2,04$ & $3,84^{\mathrm{e}}$ & 2,$95 ; 4,99$ & $3,43^{\mathrm{e}}$ & 2,$63 ; 4,48$ \\
\hline
\end{tabular}

aPor regressão logística binomial, tendo como referência a categoria ruim/muito ruim da variável dependente e a primeira categoria de resposta da variável independente.

'Ajustada por idade, faixa de renda, sexo e escolaridade.

'OR:odds ratio (razão de chances).

${ }^{d} C_{95 \%}$ intervalo de confiança de $95 \%$.

ep valor $\leq 0,05$.

Tabela 5 - Associação entre a percepção dos usuários sobre o cuidado prestado por equipes participantes do Programa Nacional de Melhoria do Acesso e da Qualidade da Atenção Básica e variáveis do atributo coordenação do cuidado, Brasil, 2012

\begin{tabular}{|c|c|c|c|c|c|c|c|c|}
\hline \multirow{4}{*}{ Variáveis } & \multicolumn{8}{|c|}{ Percepção } \\
\hline & \multicolumn{4}{|c|}{ Regular } & \multicolumn{4}{|c|}{ Muito bom/bom } \\
\hline & \multicolumn{2}{|c|}{ Análise bruta ${ }^{a}$} & \multicolumn{2}{|c|}{ Análise ajustada ${ }^{b}$} & \multicolumn{2}{|c|}{ Análise bruta ${ }^{a}$} & \multicolumn{2}{|c|}{ Análise ajustada ${ }^{\mathrm{b}}$} \\
\hline & $\mathbf{O} \mathbf{R}^{\mathrm{c}}$ & $\mathrm{IC}_{95 \%}{ }^{\mathrm{d}}$ & $\mathbf{O} \mathbf{R}^{\mathrm{c}}$ & $\mathrm{IC}_{95 \%}{ }^{\mathrm{d}}$ & $\mathbf{O R}$ & IC $_{95 \%}{ }^{d}$ & $O R^{c}$ & IC $_{95 \%}{ }^{d}$ \\
\hline \multicolumn{9}{|c|}{ Marcação de consulta com outros profissionais ou especialistas } \\
\hline Nunca & 1,00 & - & 1,00 & - & 1,00 & - & 1,00 & - \\
\hline Sim & $1,27^{e}$ & 1,$06 ; 1,51$ & $2,38^{e}$ & 2,$15 ; 2,80$ & $2,02^{\mathrm{e}}$ & 1,$70 ; 2,39$ & $5,80^{\mathrm{e}}$ & 4,$92 ; 6,84$ \\
\hline Sim, algumas vezes & 0,72 & 0,$61 ; 0,86$ & $1,60^{e}$ & 1,$35 ; 1,87$ & $0,42^{\mathrm{e}}$ & 0,$35 ; 0,50$ & $2,00^{e}$ & 1,$70 ; 2,35$ \\
\hline Não & $0,39^{e}$ & 0,$3 ; 0,46$ & $0,72^{\mathrm{e}}$ & 0,$63 ; 0,92$ & $0,14^{\mathrm{e}}$ & 0,$12 ; 0,16$ & $0,59^{\mathrm{e}}$ & 0,$42 ; 0,68$ \\
\hline \multicolumn{9}{|c|}{ Após atendimento fora da unidade de saúde, a equipe conversou sobre este atendimento } \\
\hline Não & 1,00 & - & 1,00 & - & 1,00 & - & 1,00 & - \\
\hline Sim, sempre & $2,98^{e}$ & 2,$34 ; 3,80$ & $1,85^{\mathrm{e}}$ & 1,$44 ; 2,43$ & $13,41^{e}$ & 10,$57 ; 16,91$ & $5,49^{e}$ & 4,$28 ; 7,03$ \\
\hline Sim, algumas vezes & $1,55^{\mathrm{e}}$ & 1,$22 ; 1,95$ & 1,22 & 0,$90 ; 1,54$ & $3,51^{e}$ & 2,$80 ; 4,39$ & $2,48^{e}$ & 1,$96 ; 3,13$ \\
\hline \multicolumn{9}{|c|}{ Facilidade para saber dos resultados dos exames que chegam à unidade de saúde } \\
\hline Não & 1,00 & - & 1,00 & - & 1,00 & - & 1,00 & - \\
\hline Sim, sempre & $2,78^{e}$ & 2,$44 ; 3,18$ & $2,25^{e}$ & 1,$87 ; 2,51$ & $8,89^{\mathrm{e}}$ & 7,$81 ; 10,12$ & $4,79^{\mathrm{e}}$ & 4,$09 ; 5,60$ \\
\hline Sim, algumas vezes & $1,79^{\mathrm{e}}$ & 1,$54 ; 2,08$ & $1,74^{\mathrm{e}}$ & 1,$37 ; 3,32$ & $2,40^{e}$ & 2,$07 ; 2,77$ & $2,13^{e}$ & 1,$80 ; 2,55$ \\
\hline
\end{tabular}

aPor regressão logística binomial, tendo como referência a categoria ruim/muito ruim da variável dependente e a primeira categoria de resposta da variável independente.

${ }^{b}$ Ajustada por idade, faixa de renda, sexo e escolaridade.

'OR:odds ratio (razão de chances).

d $\mathrm{C}_{95 \%}$ : : intervalo de confiança de $95 \%$.

${ }^{e} p$ valor $\leq 0,05$. 
processo assistencial, buscando conhecer e atender plenamente às necessidades de saúde dos usuários.

Cabe destacar que, no caso da avaliação externa do PMAQ-AB, pode ter ocorrido um viés de seleção, tendo em vista que os entrevistados eram usuários que já vinham recebendo atendimento na Unidade de Saúde e, portanto, tendiam a perceber mais positivamente a qualidade dos serviços. ${ }^{9}$ Além disso, as equipes aderidas ao PMAQ-AB no $1^{\circ}$ ciclo, selecionadas pelos gestores locais, podem reforçar este viés de seleção na medida em que, provavelmente, muitas se caracterizam como aquelas mais organizadas no município, o que pode refletir uma avaliação mais positiva dos usuários. Ressalta-se ainda que os resultados podem apresentar variações ao se considerar o porte populacional dos municípios que apresentam diferenças na capacidade de organização e institucionalização da ABS. ${ }^{23}$

A bibliografia existente sobre o tema da percepção de usuários sobre os serviços de saúde indica uma variabilidade de métodos e conceitos para essa aferição, sendo muito frequente se observar nos estudos elevadas taxas de

\section{Referências}

1. Ministério da Saúde (BR). Secretaria de Atenção à Saúde. Departamento de Atenção Básica. Política nacional de atenção básica [Internet]. Brasilia: Ministério da Saúde; 2012 [citado 2018 mar 28]. 110 p. (Série E. Legislação em Saúde). Disponível em: http://189.28.128.100/dab/ docs/publicacoes/geral/pnab.pdf

2. Starfield B. Atenção primária: equilibrio entre necessidades de saúde, serviços e tecnologia [Internet]. Brasilia: Unesco; Ministério da Saúde; 2002 [citado 2018 mar 28]. 726 p. Disponível em: https://www.nescon. medicina.ufmg.br/biblioteca/imagem/0253.pdf

3. Giovanella L, Mendonça MHM, Almeida PF, Escorel S, Senna MCM, Fausto MCR, et al. Saúde da família: limites e possibilidades para uma abordagem integral de atenção primária à saúde no Brasil. Ciênc Saúde Coletiva. 2009 maio-jun;14(3);783-94.

4. Haggerty J, Burge F, Lévesque JF, Gass D, Pineault R, Beaulieu MD, et al. Operational definitions of attributes of primary health care: consensus among Canadian experts. Ann Fam Med. 2007 Jul-Aug;5(4):336-44.

5. Brandão ALRBS. Avaliação da Atenção Básica pela Perspectiva dos Usuários: Adaptação do Instrumento Europep para Grandes Centros Urbanos Brasileiros - uma Aplicação na Comunidade de Manguinhos [Dissertação]. Rio de Janeiro: Escola Nacional de Saúde Pública; 2011.

6. Schembri S. Experiencing health care service quality: through patients' eyes. Aust Health Rev. 2015 Feb;39(1):109-16. satisfação ${ }^{5,24}$ Em função disso, persiste a necessidade de serem aprofundados desenhos metodológicos que consigam captar com mais exatidão os determinantes de uma melhor ou uma pior avaliação do usuário com o cuidado recebido, para que se possa conhecer e incorporar de forma mais ampla a percepção dos usuários quanto à assistência ofertada, ${ }^{6,25}$ o que contribuirá efetivamente para a organização e 0 aperfeiçoamento dos serviços de saúde.

\section{Contribuição dos autores}

Abreu DMX, Araújo LL e Matta-Machado ATG contribuíram na concepção e delineamento do estudo, organização da base de dados, análise dos resultados e sua interpretação, além da redação do manuscrito. Reis CMR, Lima AMLD, Santos AF, Jorge AO e Fonseca SD contribuíram na análise dos resultados e sua interpretação e redação do manuscrito. Todos os autores aprovaram a versão final do manuscrito e declaram ser responsáveis por todos os aspectos do trabalho, garantindo sua precisão e integridade.

7. Zastowny TR, Roghmann KJ, Cafferata GL. Patient satisfaction and the use of health services: explorations in causality. Med Care. 1989 Jul;27(7):705-23.

8. Esperidião MA, Trad LAB. Avaliação de satisfação de usuários. Ciênc Saúde Coletiva. 2005 dez;10(Supl):303-12.

9. Ministério da Saúde (BR). Secretaria de Atenção à Saúde. Departamento de Atenção Básica. Programa nacional de melhoria do acesso e da qualidade da atenção básica (PMAQ): manual instrutivo [Internet]. Brasília: Ministério da Saúde; 2012 [citado 2018 mar 28]. 62 p. (Série A. Normas e Manuais Técnicos). Disponível em: http://189.28.128.100/dab/docs/ publicacoes/geral/manual_instrutivo_pmaq_site.pdf

10. Hosmer Jr DW, Lemeshow S. Applied logistic regression. 2nd ed. New York: John Wiley \& Sons; 2000.

11. Gomes MCPA, Pinheiro R. Acolhimento e vínculo: práticas de integralidade na gestão do cuidado em saúde em grandes centros urbanos. Interface (Botucatu). 2005 ago;9(17):287-301.

12. Mishima SM, Pereira FH, Matumoto S, Fortuna CM, Pereira MJB, Campos AC, et al. A assistência na saúde da família sob a perspectiva dos usuários. Rev LatinoAm Enfermagem. 2010 maio-jun;18(3):436-43.

13. Vaitsman J, Andrade GRB. Satisfação e responsividade: formas de medir a qualidade e a humanização da assistência à saúde. Ciênc Saúde Coletiva. 2005 julset;10(3): 599-613. 
14. Girardi SN, Carvalho CL, Araújo JF, Farah JM, Wan Der Mass, L, Campos LAB. Índice de escassez de médicos no Brasil: estudo exploratório no âmbito da Atenção Primária. In: Pierantoni CR, Dal Poz MR, França T, organizadores. 0 trabalho em Saúde: abordagens quantitativas e qualitativas [Internet]. Rio de Janeiro: Cepesc/IMS/UERJ, ObservaRH; 2011 [citado 2018 mar 28]. p. 171-86. Disponível em: http://www.obsnetims.org.br/uploaded/6_10_2014__ _ livro_0_trabalho_em_saude.pdf

15. Ronzani TM, Silva CM. O Programa Saúde da Família segundo profissionais de saúde, gestores e usuários. Ciênc Saúde Coletiva. 2008 jan-fev;13(1):23-34.

16. Comes Y, Trindade JS, Shimizu HE, Hamann EM, Bargioni F, Ramirez L, et al. Avaliação da satisfação dos usuários e da responsividade dos serviços em municípios inscritos no Programa Mais Médicos. Ciênc Saúde Coletiva. 2016 set;21(9):2749-59.

17. Ramos DD, Lima MADS. Acesso e acolhimento aos usuários em uma unidade de saúde de Porto Alegre, Rio Grande do Sul, Brasil. Cad Saúde Pública. 2003 jan-fev;19(1):27-34.

18. Gouveia GC, Souza WV, Luna CF, Souza-Júnior PRB, Szwarcwald CL. Health care users' satisfaction in Brazil, 2003. Cad Saúde Pública. 2005;21(Supl 1):S109-18.

19. Moimaz SAS, Marques JAM, Saliba O, Garbin CAS, Zina LG, Adas SN. Satisfação e percepção do usuário do SUS sobre o serviço público de saúde. Physis. 2010 dez;20(4):1419-40.

\section{Abstract}

Objective: to analyze service users' perception of bealth care provided by the teams participating in the National Program for Primary Care Access and Quality Improvement (PMAQ-AB) in Brazil. Methods: this was a cross-sectional study using data from interviews with users of services provided by PMAQ-AB teams in 2012. Multinomial logistic regression was used to analyze the association between perception level (very good/good, regular, poor/very poor) and variables of four primary care attributes: accessibility, integrality, longitudinality and care coordination. Results: out of 65,391 users, $78.9 \%$ evaluated care as very good/good, $19.0 \%$ as regular and $2.1 \%$ as poor/very poor. Associations with care by the same physician (OR 3.21; 95\% CI 2.68, 3.83) and physical examination at consultation (OR 3.08; 95\% CI 2.50,3.79) were the most prominent. Conclusion: the majority of users evaluated care positively, however there is perception of care problems within the recommended attributes.

Keywords: Primary Health Care; Patient Satisfaction; Quality of Care; Cross-Sectional Studies.
20. Rosa RB, Pelegrini AHW, Lima MADS. Resolutividade da assistência e satisfação de usuários da Estratégia Saúde da Família. Rev Gaúcha Enferm. 2011 jun;32(2):345-51.

21. Almeida PF, Giovanella L, Nunan BA. Coordenação dos cuidados em saúde pela atenção primária à saúde e suas implicações para a satisfação dos usuários. Saúde Debate. 2012 jul-set;36(94):375-91.

22. Fausto MCR, Giovanella L, Mendonça MHM, Seidi H, Gagno J. A posição da Estratégia Saúde da Família na rede de atenção à saúde na perspectiva das equipes e usuários participantes do PMAQ-AB. Saúde Debate. 2014 out;38(esp):13-33.

23. Lima MADS, Ramos DD, Rosa RB, Nauderer TM, Davis R. Acesso e acolhimento em unidades de saúde na visão dos usuários. Acta Paul Enferm. 2007 mar;20(1):12-7.

24. Paiva MBP, Mendes W, Brandão AL, Campos CEA. Uma contribuição para a avaliação da Atenção Primária à Saúde pela perspectiva do usuário. Physis. 2015 set;25 (3):925-50.

25. Arruda CAM, Bosi MLM. Satisfação de usuários da atenção primária à saúde: um estudo qualitativo no Nordeste do Brasil. Interface (Botucatu). 2017 jun;21(61):321-32.

\section{Resumen}

objetivo: analizar la percepción de los usuarios sobre el cuidado prestado por los equipos del Programa Nacional de Mejora del Acceso y de la Calidad de la Atención Básica (PMAQ-AB) en Brasil. Métodos: estudio transversal, con datos de entrevistas con usuarios de los equipos evaluados por el PMAQ-AB en 2012. Se empleó regresión logística multinomial para analizar la asociación entre nivel de percepción (muy bueno/bueno, regular, malo/muy malo) y variables de atributos de la AB: accesibilidad, integralidad, longitudinalidady coordinación del cuidado Resultados: de los 65.391 usuarios, 78,9\% evaluaron la atención como muy bueno/bueno, $19,0 \%$ como regulary $2,1 \%$ como malo/ muy malo. Se destacan las asociaciones con atención por el mismo médico $\left(\mathrm{OR} 3,21 ; \mathrm{IC}_{95 \%} 2,68 ; 3,83\right)$ y realización del examen físico en la consulta (OR 3,08; $I C_{95 \%} 2,50$; 3,79). Conclusión: la mayoría de los usuarios evaluaron positivamente la atención, sin embargo existe percepción de problemas en el cuidado.

Palabras-clave: Atención Primaria de Salud; Satisfacción del Paciente; Calidad de laAtención de Salud; Estudios Transversales.

Recebido em 31/05/2017

Aprovado em 24/01/2018 